\title{
Semi-Quantitative Analysis of Iron Nodules in Equilibrium Refining Catalysts by Artificial Intelligence-Augmented Scanning Electron Microscopy
}

Ke-Bin Low ${ }^{1}$, Jian $\mathrm{Shi}^{2}$, Melissa Clough Mastry ${ }^{2}$, Vasileios Komvokis ${ }^{3}$ and Bilge Yilmaz ${ }^{2}$

${ }^{1}$ BASF Corporation, Iselin, New Jersey, United States, ${ }^{2}$ BASF Corporation, United States, ${ }^{3}$ BASF Corporation, New Jersey, United States

Fluid catalytic cracking (FCC) catalysts, in the form microspheres that contain zeolites and (silico)aluminate phases, are used in oil refining processes to convert complex and long-chain hydrocarbons into simple, useful ones, for example, gasoline, olefins, etc. Over long periods of refining, FCC catalysts will experience loss in activity. The degradation in catalytic performance can be attributed to different factors such as attrition, steam exposure, high-temperatures, time, coke formation and poisoning from metal contaminants in feedstock. Iron $(\mathrm{Fe})$ nodule formation is one characteristic manifestation of metal contamination [1,2]. Fresh catalysts will replace older ones in the FCC unit periodically in order to maintain conversion yield. The equilibrium catalysts (ECATS) are a physical mixture of fresh and aged catalysts circulating at a given time within the FCC unit.

BASF is a world-leading supplier of FCC catalysts and offers our customers value-added ECAT sample evaluation, where results will be used towards helping optimize reactor operations. Scanning electron microscopy (SEM) and energy-dispersive x-ray spectroscopy (EDS) are key techniques employed to study extent of attrition and confirm Fe nodules on FCCs. However, the characterization process can be rather lengthy, which does not align well with the quick turn-around requirements of ECAT analysis.

Artificial intelligence (AI)-augmented SEM offers a promising means to shorten turn-around time as well as track the degree of Fe contamination by automatically analyzing incoming images from the microscope based on deep-learning models trained to assess FCC microsphere attrition and Fe nodules formation on the surfaces of the FCC microspheres. Training images, containing a known mixture of fresh and Fecontaminated catalysts, were collected by SEM in backscatter mode, and subsequently used for building deep-learning models. Energy-dispersive x-ray spectroscopy was also conducted to aid model validation. Besides automatically annotating all the features in incoming images, the validated AI model can also perform semi-quantitative analysis to track the severity of Fe contamination in chronological order (Figure 1). The AI-augmented SEM approach offers the prospect of skipping tedious and lengthy EDS analysis, hence increasing analytical throughput and efficiency.

Acknowledgements:

The authors would like to thank the BASF, Iselin, New Jersey microscopy team (David Pollick, Adam Ross, Jeffrey Lin) for supplying SEM/EDS data for AI training and model validation. This work is also made possible through technical consultation with John Sosa, CEO of MIPAR Inc., on image analysis workflow design. 


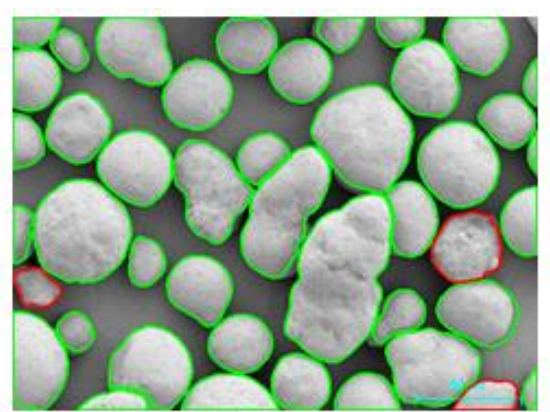

29-Oct-18

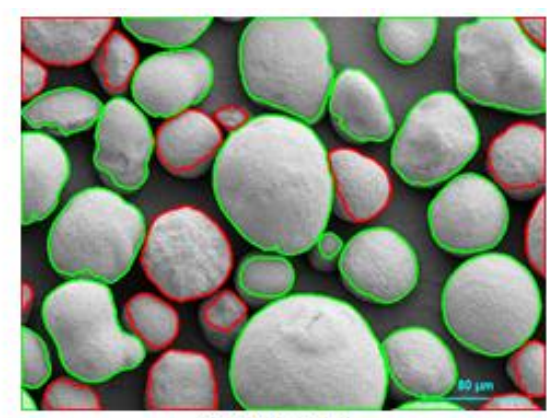

21-Nov-18

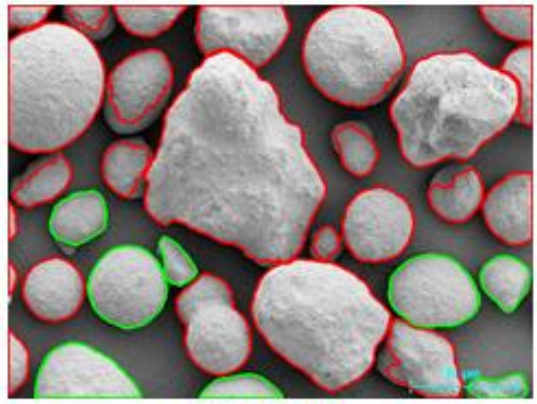

17-Dec-18

Semi-Quantitative Analysis

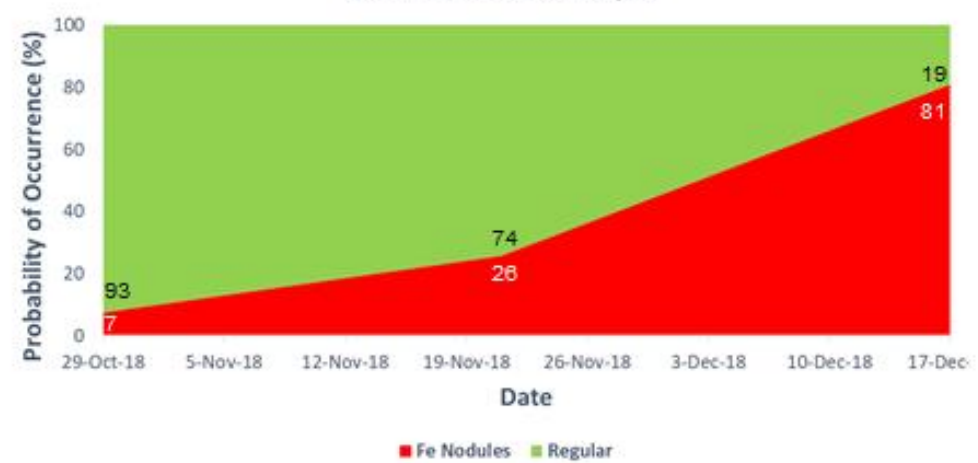

Figure 1. SEM images of chronologically-ordered ECATS (above), and the associated probabilities of Fe nodule occurrence based on the microspheres' projected area (below).

\section{References}

[1] S. Pan, L. Tan, V. Komvokis, A. Spann, M. Clough, B. Yilmaz, "Chapter 1: Nanomaterials Fueling the World" in Nanomaterials for Sustainable Energy, ACS Symposium Series (American Chemical Society, 2015).

[2] A. Shackleford, M. Clough, "Iron Poisoning Investigations Enable Opportunities for Success", Special Focus: Refining Technology Developments, Hydrocarbon Processing, September 2017. 